\title{
BreastHealth: Technological Platform for the Prevention, Detection, Monitoring and Treatment of Breast Cancer
}

\author{
María del Pilar Salas-Zárate ${ }^{1}$, Miriam Carlos-Mancilla², Emmanuel Lopez-Neri², \\ Lorena Orellana Jimenez ${ }^{3}$, Daniel Gonzalez Diaz ${ }^{4}$ \\ ${ }^{1}$ Tecnológico Nacional de México/I. T. Orizaba, Veracruz, Mexico \\ ${ }^{2}$ Centro de Investigación, Innovación y Desarrollo Tecnológico (CIIDETEC-UVM), \\ Universidad del Valle de México, Tlaquepaque, Jalisco, Mexico \\ ${ }^{3}$ INFENEFNI S.A de C.V., Zapopan, Jalisco, Mexico \\ ${ }^{4}$ Universidad Tecnológica del Centro de Veracruz, Cuitláhuac, Veracruz, Mexico \\ pilarsalaszl2@gmail.com, miriam.carlos@uvmnet.edu, \\ emmanuel.lopezne@uvmnet.edu, dralorenaorellana@hotmail.com, \\ daniel.gonzalez@utcv.edu.mx
}

\begin{abstract}
Based on the information from the World Health Organization (WHO), breast cancer is one of the most common diseases that affect women and the largest worldwide disease causing of woman mortality. Currently both, patients and health personnel use the Internet to consult medical information; specifically, patients focus on seeking support for the care of their disease. However, the efforts made to support the care of patients with this disease are not enough. Hence, this paper presents a technological platform focused on the improvement of the processes of diagnosis, medical prescription, prevention, monitoring and treatment of breast cancer, which takes advantage of innovative technologies such as collaborative filtering, semantic Web, opinion mining, Big Data, and multi-device applications. In addition, a case study is presented, and an evaluation in terms of usefulness. Finally, from the analysis of the user interviews is shown that the Web application has a high degree of acceptance of patients with breast cancer.
\end{abstract}

Keywords: big data; breast cancer; opinion mining; medical recommendation.

\section{Introduction}

The World Health Organization (WHO) published in 2018 the latest estimates on the incidence and mortality of six different types of cancer in 186 countries. According to the WHO, lung and breast cancer are the leading types worldwide in terms of the number of new cases. Based on these results, it is estimated that cancer worldwide has increased to 18.1 million new cases, while 9.6 million people will lose in 2018 the battle against this disease [1].

The cancer diagnosis in an early stage is important for patient survival. Statistically, each year, 246,660 women are diagnosed with breast cancer, of which about 40,890 results in deaths. Depending on the stage in which the disease is detected, the survival 
percentage may vary; if cancer is detected in a single breast, could reach 99 percent of survival rate; if it expands to the lymph nodes the survival rate decreases to $85 \%$; and if disseminated in different parts of the body, the 5-year rate decreases to $26 \%$. In the worst case, $5 \%$ of women are diagnosed for the first time when they are in the metastasis [2].

According to the WHO, there are three actions to improve the early stage diagnosis of cancer: 1) enhance awareness about the symptoms of cancer in the society and encourage them to seek medical attention when they detect it; 2) make investments in the equipment of health services and the training of medical personnel in order to make more accurate and timely diagnoses, and 3) ensure patients have a safe and effective available treatment neither costly nor personalized effort.

Currently, both patients and health personnel use the Internet to consult medical information without to be sure the information is accurate or true. Specifically, patients focus on seeking support for the care of their disease. However, the efforts made to support the care of patients with this disease so far are not enough.

This paper presents a technological platform focused on the improvement of the processes of diagnosis, medical prescription, prevention, monitoring and treatment of breast cancer.

Some of the benefits that this platform will provide are:

1) Guaranteed access to services and medical attention to people of any gender, personal condition, race or sexual condition, only Internet access is required.

2) Interactive exchange of opinions, an Internet interaction arises between patients, doctors or organizations through the use of social networks, forums, blogs, and open communities, among others.

3) Guaranteed and quality care.

4) Reliable medical information, the doctor supports the patient to access information reviewed/filtered by another doctor, delimiting the information available on the Web that is not relevant.

5) Patient training, the doctor offers suggestions about the patient's illness and then the patient becomes autonomous in the management of their daily health due to the process of searching for reliable information and the validation that follows with the doctor.

6) Effective time management, the patient saves time on trips for face-to-face consultations with the doctor, when doubts arise, he communicates with the doctor through his mobile device or computer.

7) Cost savings, personalized services are obtained at affordable prices. It is more expensive a private medical appointment than an online consultation.

8) Regardless of time and geographical location, the patient is guaranteed access to quality healthcare information and services.

9) Increase safety, in all stages of the medical process allowing better control in the organization of the patient information.

The rest of the paper is organized as follows. In section 2 related works discussion of the breast cancer research area are presented. In section 3 a platform architecture and main functions are described. Section 4 presents a medical specialist in oncology 
ontology. Section 5 describes and present the results of a case study. In Section 6 a web application usefulness evaluation is obtained. Finally, some conclusions and concluding remarks ideas are given.

\section{Related Work}

This section describes some of the more representative research works reported in the literature related to the breast cancer subject. For example, in [3] a mHealth Peer To Peer application to connect Hispanic patients with cancer is developed. In [4] a ubiquitous m-health system based on the user-centric paradigm of Mobile Cloud Computing (MCC) and data mining techniques are described. The core of the clientside system is developed using an Android platform, and it is used for the collection of biological data from the breast; also, a data mining technique with the Naïve Bayes (NB) classifier to predict malignancy in breast tissue and storage of MCC data on the server side is described. In [5] a method of feeling analysis is proposed to understand the emotions and opinions of users of an online support group on breast cancer related to tamoxifen.

In [6] an approach to develop a mobile web application that focuses on breast cancer patients, the proposal allows analyzing the information related to specific dietary, physical and mental aspects according to the stage of their medical treatment is presented. The authors incorporate gamification and social networks to involve and motivate people to achieve their goals of adopting healthier eating habits while increasing physical activity to ensure a lifestyle change.

In [7] a web support system for clinical decision for oncologists and patients with breast cancer is proposed. This system comprises three different forecasting methodologies: the first one is the Nottingham Prognostic Index (NPI) used clinically; second is the Cox regression model and the third one is a Partial Artificial Neural Network with Automatic Relevance Determination (PLANN-ARD). The three models produce a different prognostic index that can be analyzed together to obtain a more accurate diagnosis of the patient.

In [8] the development of a Medical Decision Support System (MDSS) for a hospital case is proposed. The authors develop the services of the medical team for cancer in hospitals from Taiwan. An ontological basis using a knowledge engineering approach for breast cancer is presented. The results show that the system improves the internal administrative efficiency, medical care quality, and the hospitals decision making.

Although the works analyzed in this section provide innovative solutions to the breast cancer domain, it is important to mention that there are no reports that address the prevention, detection, monitoring, and treatment of breast cancer. In this sense, the present work proposes a technological platform for breast cancer that takes advantage of innovative technologies such as collaborative filtering, semantic Web, opinion mining, Big Data, and multi-device applications. In the following section, the proposed platform is described in detail. 


\section{$3 \quad$ Architecture}

Figure 1 presents the general web platform architecture for the prevention, detection, monitoring, and treatment of breast cancer. The architecture is composed by four main layers, Presentation, Web services API (Application Programming Interface), Business, and Data access; each one with different modules that independently execute their tasks in order to enable the platform scalability.

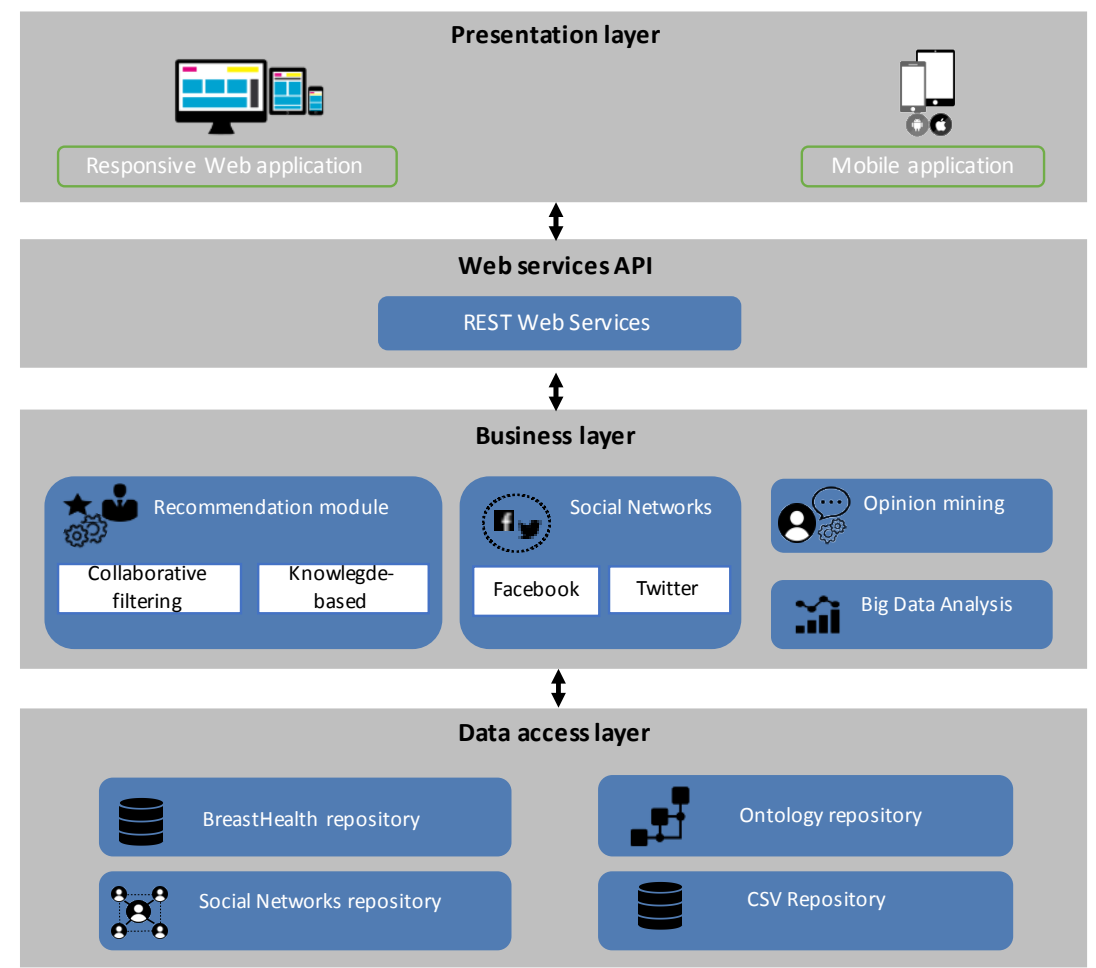

Fig. 1. Platform Architecture.

- Presentation Layer. The presentation layer enables the interaction between users and web platform, also known as a graphical user interface. The platform must be easy to use, understandable, and friendly to the user. This layer communicates with the platform through an API REST-based Web Services. Specifically, this layer covers the following applications:

- Web Application. This application enables the user access to the platform from any Web browser (Edge, Chrome, Mozilla Firefox, Safari, among others) thus enabling access from different operating systems such as Windows, Mac OS and Linux, as well as iOS, and Android. Also, a responsive User Interface approach is used, allowing the platform responds to different browsers and devices. 
- Mobile app. Consists of a mobile application for devices based on iOS and Android, being the most used versions in the market of smartphones and tablets.

- Web services API. The Web Services API will provide a set of REST-based Web services grouped by a function that will allow exchanging data between applications, in this case, between the presentation layer (Web and mobile interface) and the business layer explained bellow.

- Business Layer. The business layer will contain the main logic of data processing within the Web application. It communicates with the presentation layer to obtain the user's inputs and present the resulting information, as well to the data access layer to carry out its operations. The main features or options that the user has in their interaction with the system are:

- Recommendation module. This module will generate recommendations based on two different approaches:

○ Knowledge-Based. The recommendations generated by this module are based on the knowledge we have about the items (oncologists and hospitals) that the user has valued (either implicitly or explicitly).

○ Based on collaborative filtering. The recommendations generated by this module will be based on similarities between the active user (the user to be recommended) and the rest of the users of the system. The items (hospitals and oncologists) will be recommended by those that have not been rated by the active user and that have been well evaluated by similar users.

- Social networks Integration. This module enables opinions collection from oncologists and hospitals from social networks like Facebook and Twitter.

- Opinion mining. Enables user opinions analysis regarding oncologists and hospitals from both the web platform and social networks like mentioned before. This analysis allows the generation of recommendations based on user opinions.

- Big Data Analysis. This module considers the user data analysis available in the repositories of the platform. In addition, an analysis of large volumes of information (Big Data) will be carried out. User behavior patterns will be obtained to be useful for recommending treatments, diagnoses or follow-up the conditions not only based on similarity or previous clinical history experiences but according to the user's particular behavior patterns depending on the type of condition of the cancer. If the disease is in its terminal phase, its state of mind and other social factors acquired from medical social networks.

- Data access layer. Enables simplified the access to the stored data founded in this layer, which is comprised of the main logic of access and persistence of data within the Web application. This layer will have to support the storage of data, the recovery of information, and the concurrence of multiple users accessing the information. The stored information in this layer is:

- Breast Health repository. This repository will contain information related to recommendations, clinical history, and profiles of physicians and patients registered in the platform.

- Social networks Repository. Contains opinions of registered oncologists and hospitals on the platform from social networks such as Facebook and Twitter. 
- Ontology repository. Used for storing and managing access to the information and knowledge based on an OWL (Web Ontology Language) format, such as, the ontology models, the oncology specialist data, and the breast cancer clinical presentation.

- CSV Repository. This repository contains CSV files (simple text with commas separated values). These files allow representing the matrix scheme of the system in which the pertinent data are stored to make medical recommendations.

\section{$4 \quad$ Medical Specialist Ontology}

Since the purpose of this paper is to model the more representative oncology specialists, such as hematologist-oncologist, gynecologist-oncologist among others. We have designed an ontology of medical specialist in oncology. The proposed ontology is based on the web ontology language (OWL). The complete ontology is shown in Fig.2. The main class of the specialist is composed of Medical Specialty, General medicine, and General practitioner. The first one is subdivided into gynecologist, hematologist, oncologist, orthopedist, pathologist, and radiologist.

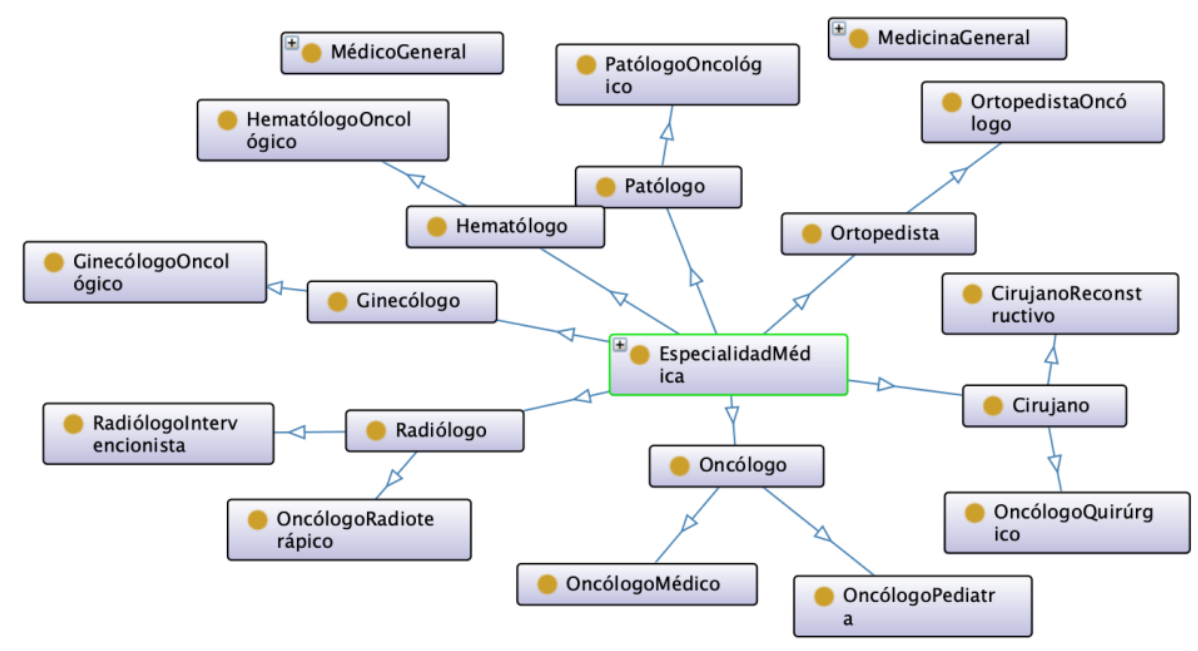

Fig. 2. Medical Specialist Ontology.

The Menthonlogy method presented in [9] was followed for the design of the ontology of medical specialists. Specifically, a series of activities were carried out, each of which focuses on one aspect of the conceptual model of knowledge: terms, taxonomy, relationships, axioms, and rules.

\section{Case Study: Breast Cancer Patients' Recommendations}

To this case study, the monitoring of the health status of breast cancer patients is based on the following conditions: 
1. The user is able to supervise its activities, habits, symptoms, and vital signs for the purpose of learning and evaluate its state of health.

2. The user is looking for health's recommendations in order to stay as healthy as possible to reduce the possibilities incidence of breast cancer.

3. The user seeks to know the best oncologist experts for its kind of breast cancer according to its city, also the best options for hospitals in the breast cancer treatment.

A possible solution for any of these previous situations are solved using the BreastHealth platform. BreastHealth allows any user to register and consults symptoms, habits, and vital signs. The user will be able to visualize medical recommendations to improve and maintain its health. Furthermore, the platform allows to search hospitals based on its treatments and search medical specialists in breast cancer.

\subsection{Medical Recommendations for Breast Cancer Patients}

BreastHealth allows monitoring any habits, symptoms, and vital signs as a fundamental part of the platform to provide good recommendations. Every user is responsible to keep update its own information about any stride and setbacks during any treatment in order to provide a useful and personalized information; such information is useful to the system and the medical specialist who is giving recommendations.

Medical recommendations make easier for patients to keep control over diverse health aspects. The first recommendations to consider are those related to daily routines, since, through these it is possible to control the affectations that directly or indirectly impact on the vital signs of the users causing symptoms that are harmful to their health.

Fig. 3 shows some recommendations provided by the system for user habits, which, if carried out, will reduce or maintain the negative impact they have on the users' health, particularly reflected through symptoms that can be registered by the users. To obtain the desired information, it is only necessary to enter the menu "Habits". As can be seen, the system provides habit recommendations by date.

\subsection{Recommendations of Hospitals and Oncologists}

The user has the possibility to search an oncologist by name, state, and specialty. Once the search has been made, the results will be displayed and the user will be able to visualize and consult every doctor resume and entire information on a detail screen. In this section, the system will deploy medical specialist recommendations according to the user preferences (see Fig. 4). With regards to hospitals, the system acts just like the medical specialist section and display hospitals recommendations according to the user preferences. 


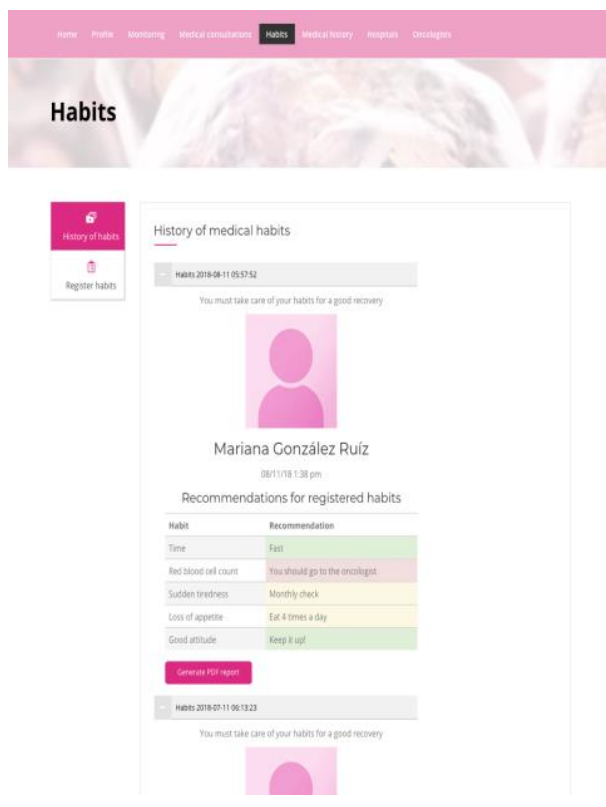

Fig. 3. Medical recommendations.

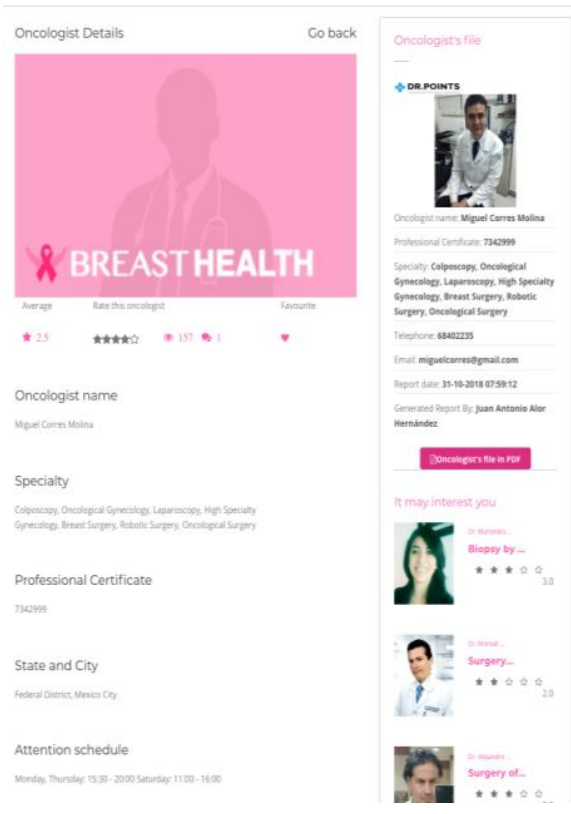

Fig. 4. Recommendation of oncologists

\section{$6 \quad$ Results}

The evaluation method is based on the satisfaction metrics of the user defined on SQuarRE ISO /IEC 25010: 2011 (International Standard Organization 2011). This standard ISO / IEC 25010: 2011 define two models of quality composed of general software characteristics; these features are composed of sub-general characteristics and specific attributes.

In the quality framework defined in ISO/ IEC 25010: 2011, user satisfaction is related to the grade of use of a system or product in a specific context. This model is composed of sub-characteristics such as usefulness, trust, satisfaction, and conform. In this proposal is only used the usefulness sub-characteristic to use a unified evaluation of the support system during the decision making. The usefulness is formally defined as the grade of client satisfaction in the goal fulfillment.

To evaluate the usefulness sub-characteristic, there are used four questions of the evaluation usability framework defined in ResQue [10] (Table 1). ResQue is a framework focused on the user experience with recommendation and decision making systems; which consist of constructions, metrics, and questions categorized by four dimensions (perceived system qualities, users' beliefs, subjective attitudes, and behavioral intentions.). Particularly, the ResQue usefulness is defined as a constructor and a metric named "user acceptance" dimension. This dimension allows grading two aspects: decision quality and the decision support through the four questions presented in Table 1. 
Every answer of the questions must have a value 1 "Very disagree" to 5 "Strongly agree" in the rating scale of Likert according to ResQue. The questionnaire was applied to 20 people with breast cancer and they used the web application during a period of 10 days.

Table 1. Usefulness questions.

\begin{tabular}{ll}
\hline ID & Question \\
\hline Q1 & $\begin{array}{l}\text { The recommended elements effectively helped to find the indicated hospitals and } \\
\text { medical specialist (decision quality) }\end{array}$ \\
Q2 & $\begin{array}{l}\text { The recommended elements certainly influenced the selection of hospitals and } \\
\text { medical specialist (decision quality) } \\
\text { The platform provides the necessary support to find the indicated hospitals and } \\
\text { medical specialist for the breast cancer treatment (decision support) } \\
\text { The platform provides the necessary support to select the indicated hospitals and } \\
\text { medical specialist for the breast cancer treatment (decision support) }\end{array}$ \\
Q4
\end{tabular}

According to Fig. 5., we are able to obtain the next conclusions: between 16 and 20 participants consider the web system as helpful, they were able to find recommendations for hospitals and medical specialist (Q1). Between 15 to 20 participants consider the recommended elements really affected during the selection of hospitals and cancer specialist (Q2). Between 17 to 20 participants were agreed in the platform provides the necessary support to find hospitals and cancer specialist according to different cancer medical treatments (Q3). Finally, between 16 to 20 participants consider the platform as useful to find locations of hospitals and cancer specialist for the cancer treatment $(\mathrm{Q} 4)$.

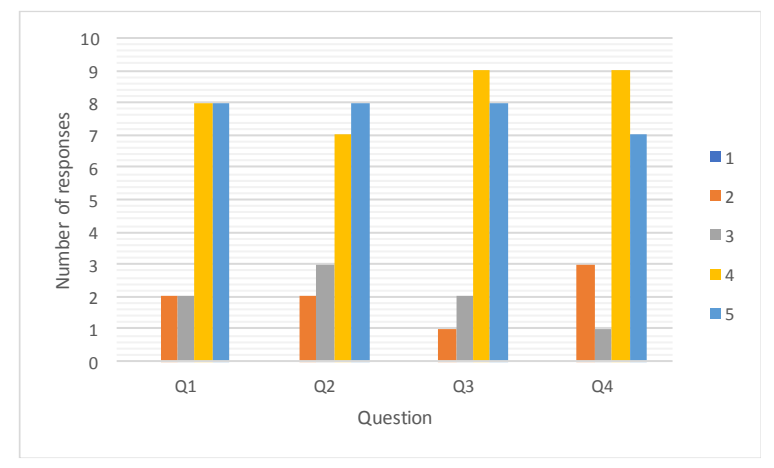

Fig. 5. Evaluation results.

\section{Conclusions}

A platform to support medical decision making to prevent, diagnose, treatment and monitoring of breast cancer is presented. This tool takes advantage of new technologies and innovations such as collaborative filtering, semantic web, opinion mining, big data, and multi-device applications. A case study was presented and it proves that Breast- 
Health is easy to use, and the generated information is useful to the users such as patients and medical specialist enrollees in the platform who gives services to cancer patients.

In this work, an ontology that identifies medical specialists in Oncology is implemented. This ontology allows identifying the adequate medical specialist for monitoring and evaluation of every patient. The platform also provides medical recommendation related to daily routines, since, through these it is possible to control the affectations that directly or indirectly impact on the vital signs of the users causing symptoms that are harmful to their health

The web application was evaluated through usefulness terms. The obtained results show a high grade of acceptance between the main users of this proposal, breast cancer patients; the web application will be improved in a near future to bring a better treatment to users from specialists.

Acknowledgments. The authors are grateful to Mexico's National Council of Science and Technology (CONACYT) for supporting this work.

\section{References}

1. OMS: Las 10 principales causas de defunción.

2. Cancer.Net: Cáncer de mama: Estadísticas | Cancer.Net.

3. Banas, J.R., Victorson, D., Gutierrez, S., Cordero, E., Guitleman, J., Haas, N.: Developing a Peer-to-Peer mHealth Application to Connect Hispanic Cancer Patients. J. Cancer Educ. 32, pp. 158-165 (2017)

4. Gatuha, G., Jiang, T.: Android Based Naive Bayes Probabilistic Detection Model for Breast Cancer and Mobile Cloud Computing: Design and Implementation. Int. J. Eng. Res. Africa 21, pp. 197-208 (2015)

5. Cabling, M.L., Turner, J.W., Hurtado-de-Mendoza, A., Zhang, Y., Jiang, X., Drago, F., Sheppard, V.B.: Sentiment Analysis of an Online Breast Cancer Support Group: Communicating about Tamoxifen. Health Commun. 33, pp. 1158-1165 (2018)

6. Economou, D., Dwek, M., Roberston, C., Elliott, B., Kounenis, T., Azimi, T., Ramezanian, M., Bell, N.: PhytoCloud: A Gamified Mobile Web Application to Modulate Diet and Physical Activity of Women with Breast Cancer. In: 2017 IEEE 30th International Symposium on Computer-Based Medical Systems (CBMS). pp. 684-689. IEEE (2017)

7. Fernandes, A.S., Alves, P., Jarman, I.H., Etchells, T.A., Fonseca, J.M., Lisboa, P.J.G.: A Clinical Decision Support System for Breast Cancer Patients. Presented at the (2010)

8. Liao, S.H., Kan, S.L., Lu, S.: The Implementing of an Ontology-Based Medical Decision Support System on Breast Cancer. In: International Conference on Artificial Intelligence and Software Engineering (AISE). pp. 220-235 (2014)

9. Fernández-López, M., Gómez-Pérez, A., Juristo, N.: METHONTOLOGY: From Ontological Art Towards Ontological Engineering. Proc. Ontol. Eng. AAAI-97 Spring Symp. Ser. AAAI-97 Spring Symp. Ser. 24-26 March 1997. Stanford Univ. EEUU (1997)

10. Pu, P., Chen, L., Hu, R.: A user-centric evaluation framework for recommender systems. In: Proceedings of the fifth ACM conference on Recommender systems - RecSys '11. p. 157. ACM Press, New York, New York, USA (2011) 